Check for updates

Cite this: Soft Matter, 2018, 14, 3057

Received 30th January 2018, Accepted 25th March 2018

DOI: $10.1039 / c 8 s m 00224 j$

rsc.li/soft-matter-journal

\section{Generation of 3-dimensional multi-patches on silica particles via printing with wrinkled stamps $\dagger$}

\author{
D. John, ${ }^{a}$ M. Zimmermann (D) bc and A. Böker (D) *bc \\ A simple route towards patchy particles with anisotropic patches with respect to a different functionality \\ and directionality is presented. This method is based on microcontact printing of positively charged \\ polyethylenimine (PEI) on silica particles using wrinkled stamps. Due to the wrinkled surface, the number \\ of patches on the particles as well as the distance between two patches can be controlled.
}

The design of complex structures with specific functionalities via self-assembly plays an important role in nature (i.e. DNA, biomimetic structures, etc.). However, the transfer of these self-assembly concepts to create complex synthetic materials poses a challenge as the underlying mechanisms are not yet completely understood and respective programmable structures not always easy to get. A promising approach towards artificial self-assembly is the use of multivalent micro- and nanoparticles (i.e. patchy particles), which can assemble to complex but defined structures such as capsules, networks or other 2D and 3D structures via interparticle interactions. ${ }^{1,2}$ Such particles and the respective assembled structures have potentials especially in the fields of drug delivery, optoelectronics or biosensoring. ${ }^{3,4}$

In the last few years, researchers gained more control over the synthesis of patchy particles opening opportunities to adjust properties such as functionality, interparticle interaction and directionality. ${ }^{5}$ These are the main properties that later on direct the kind of self-assembly and the structure that result. To date, quite a number of methods such as colloidal assembly, ${ }^{6}$ microfluidics, ${ }^{7,8}$ templating, ${ }^{9,10}$ one-pot synthesis, ${ }^{11}$ nanosphere ${ }^{12}$ and particle lithography ${ }^{13}$ as well as glancing angle deposition ${ }^{14}$ are available to yield patchy particles. ${ }^{15}$ Additionally, microcontact printing $(\mu \mathrm{CP})$ becomes an increasingly important method to generate patchy or Janus particles. ${ }^{16-19}$ The advantage of this method over most of the other procedures is the preparation of

\footnotetext{
${ }^{a}$ Saint Gobain Glass Deutschland, Poststraße 103, 51143 Köln, Germany. E-mail: daniela.john@saint-gobain.com

${ }^{b}$ Fraunhofer-Institut für Angewandte Polymerforschung IAP, Geiselbergstraße 69, 14476 Potsdam-Golm, Germany. E-mail: marc.zimmermann@iap.fraunhofer.de, alexander.boeker@iap.fraunhofer.de

${ }^{c}$ Lehrstuhl für Polymermaterialien und Polymertechnologien, Universität Potsdam, Karl-Liebknecht-Straße 24-25, 14476 Potsdam, Germany

$\dagger$ Electronic supplementary information (ESI) available: Presenting synthesis, general procedures and further results. See DOI: 10.1039/c8sm00224j
}

particles with an asymmetric geometry and/or different functionalities of the patches.

$\mu \mathrm{CP}$ on flat surfaces was first described by Kumar and Whitesides in $1993 .{ }^{20}$ Their aim was a fast and easy way to replicate complex patterns avoiding the use of classical lithography. In general, $\mu \mathrm{CP}$ relies on the selective transfer of inks from a patterned and elastic stamp onto a substrate while not being limited to a few particular systems. ${ }^{21,22}$ Depending on the modification of stamps and surfaces, silanes, ${ }^{23,24}$ lipids ${ }^{25}$ proteins, ${ }^{26,27}$ dendrimers, ${ }^{28}$ DNA, ${ }^{29}$ polymers ${ }^{17}$ and nanoparticles ${ }^{30}$ can be transferred. The driving force concerning the printing of inks from a stamp onto a substrate surface is mainly a higher affinity of the ink to the substrate than to the stamp. ${ }^{31}$ In order to firmly bind the transferred ink to the respective substrate, a lot of examples for the reactions have been utilized for $\mu \mathrm{CP}$ on surfaces: coppercatalyzed cycloaddition of alkynes and azides, ${ }^{32,33}$ epoxy ring opening, ${ }^{16,34}$ imine formation of amines and aldehydes, ${ }^{17,35}$ Diels-Alder reactions ${ }^{36}$ and thiol-ene click chemistry. ${ }^{33,37}$

In comparison to printing onto flat substrates, which is a well-established procedure, printing onto particles is a rather new and more complex field and so far, only some groups try to use polymer and silica particles as a substrate. ${ }^{16,17,23,26}$ A special type of printing is the so-called "sandwich" printing. This allows the simultaneous printing on the two hemispheres of the particle as well as the use of different inks to generate anisotropic particles. ${ }^{16,18,19,33,38}$

Polydimethylsiloxane (PDMS) is the most common material to create stamps due to its flexibility, transparency and stability, which are important features for $\mu \mathrm{CP}$. At the same time, PDMS substrates are susceptible to controlled wrinkling of their surfaces, which can be used as a low-cost alternative to lithographic methods to generate tailor-made nanostructured templates or stamps. By using wrinkled substrates as stamps, we aim to synthesize multi-patch particles with the opportunity of tuning the size 
and the number as well as the distance between the patches on the particles. To date, wrinkled stamps have only been used for printing onto flat substrates. ${ }^{39}$ In the following, we show that it is also possible to print onto silica particles with wrinkled stamps in order to create particles with more than one patch, a more complex and asymmetric geometry of these patches as well as different functionalities. Note that in the case of printing with wrinkled stamps, the printing mechanism differs compared to printing with flat stamps, where we deal with the known $\mu \mathrm{CP}$ process. With wrinkled stamps, the ink is transferred from the grooves of the wrinkled surface to the substrate, which is called intaglio printing. ${ }^{40}$

Wrinkling of surfaces typically results from a stress release of a bilayer system consisting of a thin stiff layer on an elastic fundament. $^{41,42}$ The wrinkle parameters such as periodicity $(\lambda)$ and amplitude $(A)$ can be tuned by the thickness of the stiff layer and the elasticity of the substrate.

Having this in mind, a wrinkled structure on the PDMS surface can be generated by treating a stretched PDMS substrate with air plasma (see the ESI; $\dagger$ Fig. S1A) followed by the release of applied stress. The higher the plasma dose (product of plasma treatment time and power), the thicker and stiffer the $\mathrm{SiO}_{x}$ layer on top of the PDMS and in turn the broader $\lambda$ and a higher $A .^{43}$ However, as will be shown also in this contribution, not only $\lambda$ and $A$ affect the printing process but also the stiffness of the stamp. Unfortunately, wrinkled surfaces with different feature dimensions also come along with different stiffnesses, which indicates that two influential parameters would be changed at the same time. Fortunately, this issue can be rectified using replicas of the original wrinkled structure. These replicas have no oxidized top layer that is crucial for the wrinkling process and thus can consist of uniform materials (see the ESI; $\dagger$ Fig. S1B and C).

In this contribution, we demonstrate the applicability of the proposed process towards complex patchy particles and investigate the influence of stiffness and wavelength of the stamps, the concentration of the ink as well as the particle size and solvent on the printing step, and the final structure of the patches on the particles.

In contrast to common printing techniques on flat or spherical substrates, the geometry and number of the printed patches can be varied when using wrinkled stamps. Apart from this, the procedure of the printing process proceeds analogously to classical printing techniques with flat stamps onto spherical particles (Fig. 1). ${ }^{16,26}$ The printing process commenced by generating a particle monolayer on top of a glass substrate by spin-coating or drop-casting. In parallel to that, plasma-activated wrinkled stamps were covered with an aqueous PEI solution. We used polyethylenimine (PEI) as the ink, as this positively charged polyelectrolyte can electrostatically interact with the negatively charged silica surface of the particles. After a drying step, the loaded stamps were pressed onto the fixed particle monolayer for 5 to 10 seconds. Finally, the particles were removed from the stamps by ultra-sonication in ethanol or acetone. For the fluorescence measurements, the PEI patches were additionally marked with the fluorescent dye fluorescein isothiocyanate (FITC).

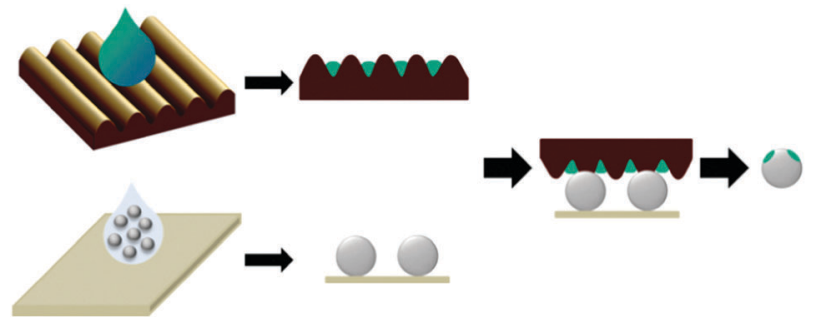

Fig. 1 Schematic depiction of the intaglio printing process: loading of the stamps with ink, generating a particle monolayer on a glass slide, and printing from one side and removing particles from the stamp.

The chosen wrinkle wavelengths of around $1.5 \mu \mathrm{m}$ and $3 \mu \mathrm{m}$ provide a better resolution on the optical microscope and generally allow an easier handling of the printing process compared to the features in a nanometer range. For the particles, a diameter of $5 \mu \mathrm{m}$ was firstly chosen. Here, we expected two to three patches on top of the particles after the printing owing to the specific relative dimensions of particles and wrinkles. Furthermore, all of the patches should have a distance between each other that reflects the chosen wavelength of the stamp.

The first observation that was made is that a change in the wavelength of the wrinkled stamps alters the distance between two of the resulting patches on the particle surface. This is illustrated in the ESI $\dagger$ (Fig. S5), where the size ratio between particle diameter, wavelength and patch geometry is shown. Initially, we printed with the original wrinkled stamps for the first experiments. Original specimens with different wavelengths, as mentioned before, also exhibit a different surface stiffness originating from a varying thickness of the oxide layer on top of the substrate due to a different plasma treatment time. While generating two patches with $1.5 \mu \mathrm{m}$ wrinkles is possible (see Fig. 2A and B), two separate areas are visible on top of the particles. The distance between both patches is, as expected from the stamp dimensions, 1.3-1.5 $\mu \mathrm{m}$. On the other hand, printing with $3 \mu \mathrm{m}$ original wrinkles leads to nearly no patches on the particles. This insufficient transfer of the ink is attributed to the relatively high stiffness of this stamp. When using a harder stamp, the reaction area between the stamp and the particle is relatively low due to a lower flexibility of the stamp in comparison to stamps with a smaller wavelength. In comparison to flat stamps, the reaction area during the printing process is already significantly decreased due to the wrinkled structure of the stamps. The additional decrease due to a stiffness increase leads to no sufficient contact between the stamp and the particle so that no ink is transferred. This correlation between the reaction area, patch size and stiffness of the stamp was also described by Jiang and Granick in $2009 .^{23}$

Two measures were tested in order to enable us to also print with stamps with wavelengths equal to or higher than $3 \mu \mathrm{m}$ : the use of cast wrinkles as stamps (PDMS replicas) as well as the change in the solvent for the release of the particles from the stamps after printing. The first approach limits the stiffness of the cast stamps keeping it independent of the wavelength; the second approach uses the varying solubility of PEI in protic (here: ethanol) and aprotic (here: acetone) solvents. 

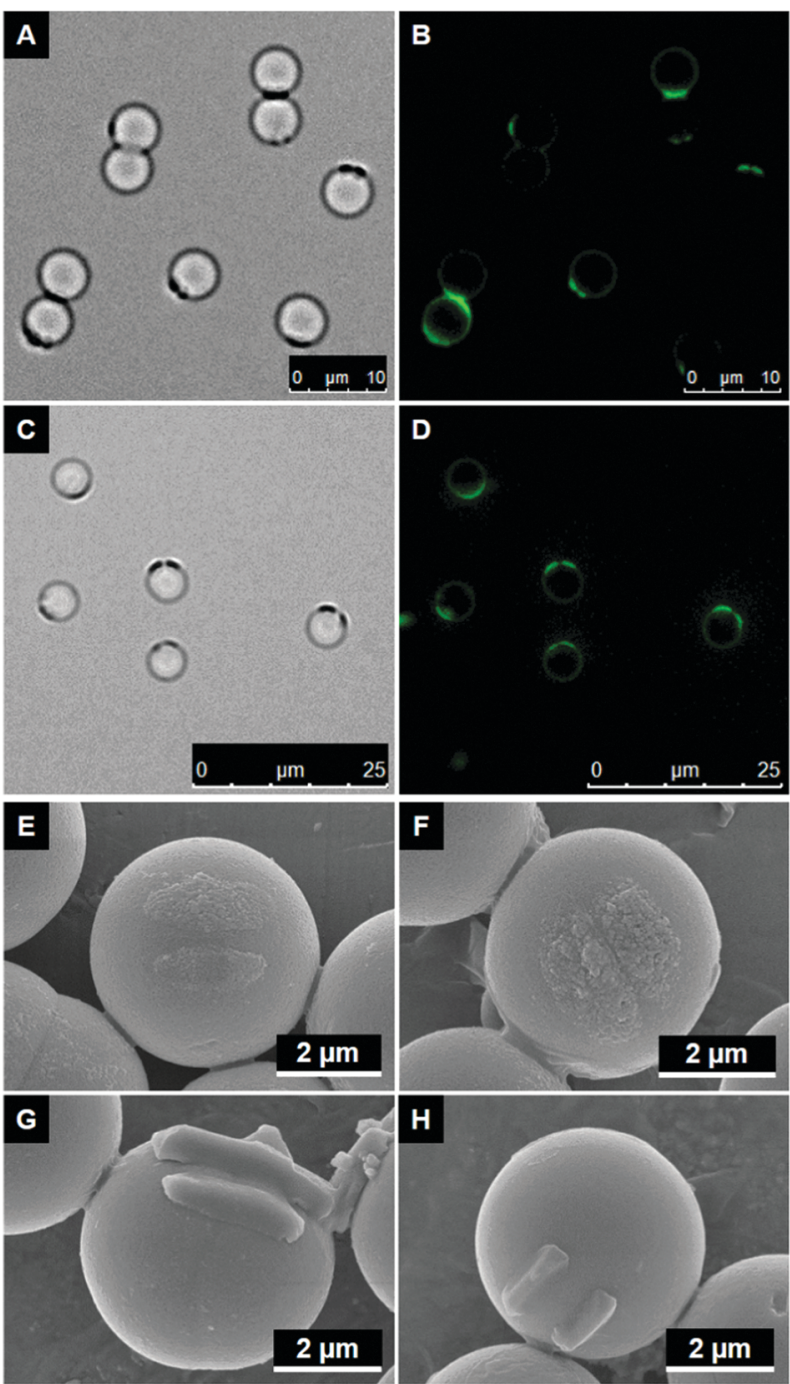

Fig. 2 (A-D) Bright field and fluorescence images of silica particles $(5 \mu \mathrm{m})$ after printing with original stamps with a wavelength of $1.5 \mu \mathrm{m}(\mathrm{A}+\mathrm{B})$ and with cast stamps with a wavelength of $3 \mu \mathrm{m}(C+D)$. Particles were removed with ethanol and patches were labeled with FITC. (E and F) SEM images to compare patch size after printing with original wrinkle stamps (A) and cast wrinkle stamps (B) with 4 wt\% PEl. Particles were removed with ethanol. ( $G$ and H) SEM images of silica particles $(5 \mu \mathrm{m})$ after printing with stamps with a wavelength of $1.5 \mu \mathrm{m}$ and PEI concentrations of $4 \mathrm{wt} \%$ (C) and $2 \mathrm{wt} \%$ (D). In this case, particles were removed by acetone.

By using cast wrinkles with this higher wavelength of $3 \mu \mathrm{m}$, printing onto silica particles becomes possible (Fig. 2C and D), because enough ink is transferred along the reaction area due to a much lower stiffness of the stamp. It is noted that for both wavelengths that have been tested at this point $(1.5 \mu \mathrm{m}$ and $3 \mu \mathrm{m}$ ), we recognize that there are particles with mostly two patches but occasionally also with only one patch. This observation originates from the fact that the small distance between two reaction areas, i.e. the area where the ink is transferred from the stamp to the particle, is lower than the wavelength of the wrinkled stamp. In some cases, the low distance between the patches is completely vanished due to the pressure during the printing process in combination with the deformation of the flexible stamp. SEM images, as presented in Fig. $2 \mathrm{E}$ and $\mathrm{F}$, describe the differences between the use of an original and a cast stamp and also show the resulting distance between two patches. Comparing original and cast stamps reveals the influence of the stiffness of the stamp in a very obvious way. The patches created by using the original stamp (Fig. 2E) are smaller; the gap between them is around $360 \mathrm{~nm}$ wide. Compared to that, the gap between the patches created by using the cast stamp is so low that it is nearly invisible. Yet, the mean distance between the patches in both cases correlates well with an used stamp wavelength of $1.5 \mu \mathrm{m}$.

At this point, we had always used ethanol for removing the printed particles from the stamps. However, the second solution to print the patches with $3 \mu \mathrm{m}$ stamps was changing the solvent. Instead of using the protic solvent ethanol it appeared reasonable to also test an aprotic solvent like acetone, which is explained in the following.

The adsorption of polyelectrolytes onto surfaces depends on several parameters: interactions between polyelectrolytes and substrates as well as between polyelectrolytes and solvents, $\mathrm{pH}$, charge density, temperature, surface roughness and adsorption time. ${ }^{44}$ In particular, the interaction between the polyelectrolyte and the solvent, in other words the solubility of the polyelectrolyte, influences the thickness of the adsorbed layer on the substrate. ${ }^{4,46}$ Indeed, the thickness of the patches in our case correlates well with the solubility of the polyelectrolyte in the surrounding medium and its interaction with the substrate. The solubility of PEI is higher in protic solvents than in aprotic solvents. Therefore, PEI adopts a more coiled conformation in acetone, which leads to thicker adsorbed patches, because the PEI film under the particle is removed entirely. ${ }^{45}$ Although we did not observe detachment of patches during the use of ethanol as the solvent, the possibility of slight removal, and therefore thinning of the patch, persists.

By comparing Fig. 2E and F with Fig. $2 \mathrm{G}$ and H, we also recognize a difference in the patch appearance upon solvent change apart from the increase in thickness. The particles removed with ethanol show not only a thinner but also a less defined structure, whereas the particles removed with acetone have a much more spatially defined structure resembling the form of the amplitude of the wrinkled stamps. This is also a further indication for an intaglio printing process, as not only do the patches adopt the structure of the wrinkle grooves but also does the patch height correlate very well with the amplitude of the used stamp.

Fig. $2 \mathrm{G}$ and $\mathrm{H}$ also underline the influence of the ink concentration. We normally use an aqueous solution of PEI with a concentration of $4 \mathrm{wt} \%$ for the printing process (Fig. 2G); however, a concentration of $2 \mathrm{wt} \%$ has also been tested (Fig. $2 \mathrm{H}$ ). Comparing the patches on the particles manufactured with varying concentrations, it turns out that the patch size increases with increasing ink concentration. Hence, the patch size depends on both the ink concentration and the amplitude of the stamp.

For a much easier handling and characterization of the particle surface, larger silica particles with a diameter of $20 \mu \mathrm{m}$ have been utilized. First experiments with these larger particles 
showed that the best results could be achieved with cast wrinkles as stamps and acetone as the solvent, the same result we achieved for the smaller particles before. For this reason, we are concentrating on these results in the following part. Just as for the particles with a diameter of $5 \mu \mathrm{m}$, we used wrinkled stamps with a wavelength of $1.5 \mu \mathrm{m}$ or $3 \mu \mathrm{m}$. Printing the stamps with these wavelengths onto larger particles leads to the generation of an increased number of patches on the particle surface.

The use of wrinkle stamps with a wavelength of $3 \mu \mathrm{m}$ should result in at least three patches, whereas wrinkled stamps with a wavelength of $1.5 \mu \mathrm{m}$ should lead to even five or more patches (see the ESI, $\dagger$ Fig. S5). The printing process itself remains unaltered.

A clear surface modification with PEI is also achieved with the large particles when using cast wrinkle stamps (Fig. 3A to F). A further magnification of single particles reveals a striped structure on the respective particle surface (Fig. $3 \mathrm{~B}+\mathrm{C}$ and $\mathrm{E}+\mathrm{F}$ ). The stamps with a wavelength of $1.5 \mu \mathrm{m}$ lead to seven to eight patches (see Fig. 3A-C) while the stamps with a wavelength of $3 \mu \mathrm{m}$ lead to three to five patches (see Fig. 3D-F). The reason for obtaining more than the expected patch number is the elasticity of the stamp in combination with the pressure applied during the printing process. These factors were not considered for the calculation of the theoretical patch number, which used only the dimensions of particle and wavelength. During printing, the stamp is able to adjust to
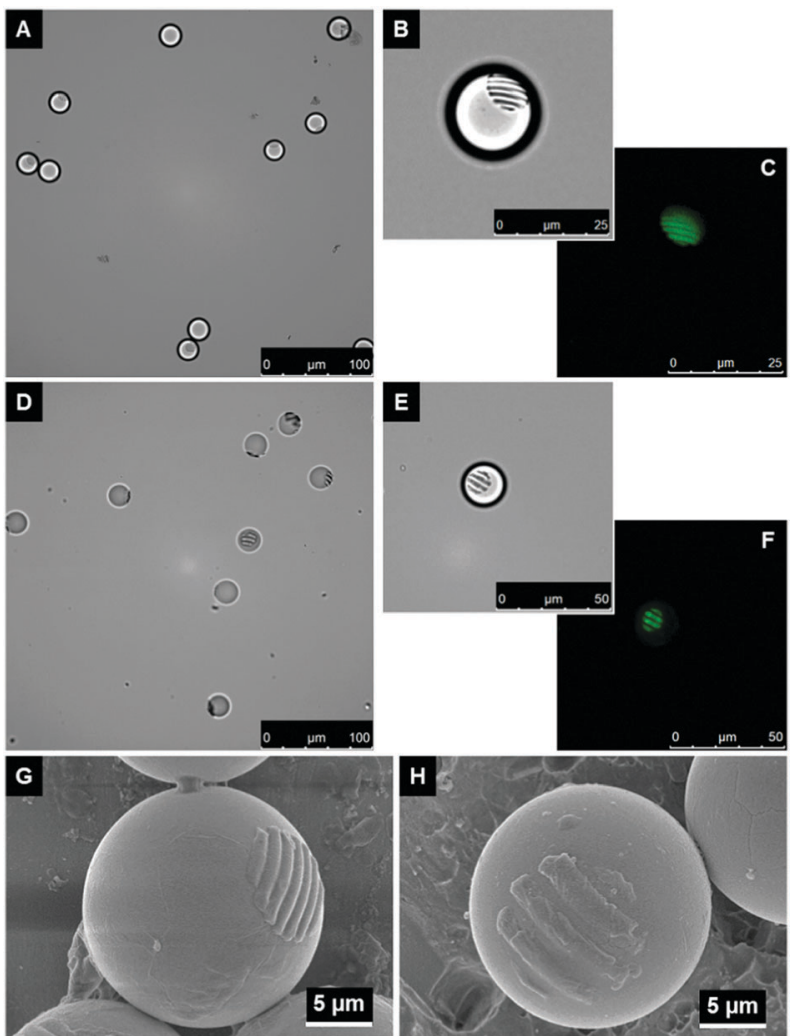

Fig. 3 (A-F) Bright field and fluorescence images of silica particles $(20 \mu \mathrm{m})$ after printing with cast wrinkle stamps with wavelengths of $1.5 \mu \mathrm{m}$ (A-C) and $3 \mu \mathrm{m}$ (D-F). (G and H) SEM images of silica particles (20 $\mu \mathrm{m})$ after printing with wrinkled stamps with wavelengths of $1.5 \mu \mathrm{m}(\mathrm{G})$ and $3 \mu \mathrm{m}(\mathrm{H})$. $\mathrm{PEI}$ concentration was set to $4 \mathrm{wt} \%$, particles were removed by acetone, and patches were labeled with FITC. the form of the particle surface whereby the entire reaction area increases.

Fig. 3(I and J) underline the results of fluorescence microscopy. The presented SEM images show the different patch numbers when using stamps with wavelengths of $1.5 \mu \mathrm{m}$ (Fig. 3G) and $3 \mu \mathrm{m}$ (Fig. 3H), respectively. Other parameters such as the thickness of the patches are again influenced by the ink concentration. Therefore, the differences between printing on smaller and larger particles are mainly manifested in a varying patch number. Ink concentration and solvent have similar effects on the patch formation.

As a final step, we demonstrate the implementation of our printing concept in a sandwich printing process, enabling the printing of differently functionalized patches simultaneously on both particle hemispheres. When the two particle hemispheres are supposed to be modified with different functionalities (here: different fluorescent dyes), one has to functionalize the ink prior to the printing in contrast to the previously applied procedure, as a post-print functionalization would only allow for an attachment of one type of dye on both hemispheres, hence leading to the same fluorescent color for both particle sides. For this reason, we modify PEI prior to printing with the fluorescent dyes fluorescein isothiocyanate (FITC) and rhodamine B isothiocyanate (RBITC) respectively. The modified PEI (PEI-FITC or PEI-RBITC) can directly be used as the ink in which the loading process is the same as for the unmodified PEI, which was already described.

The depiction of the "sandwich"-printing technique, which is just a modification of the intaglio printing process from one side, is shown in Fig. 4A. Here again, we only use the cast wrinkle stamps to rule out the influence of an altering wrinkle stiffness. The first particle hemisphere is printed as described in Fig. 1. After that step and the removal of the stamp together

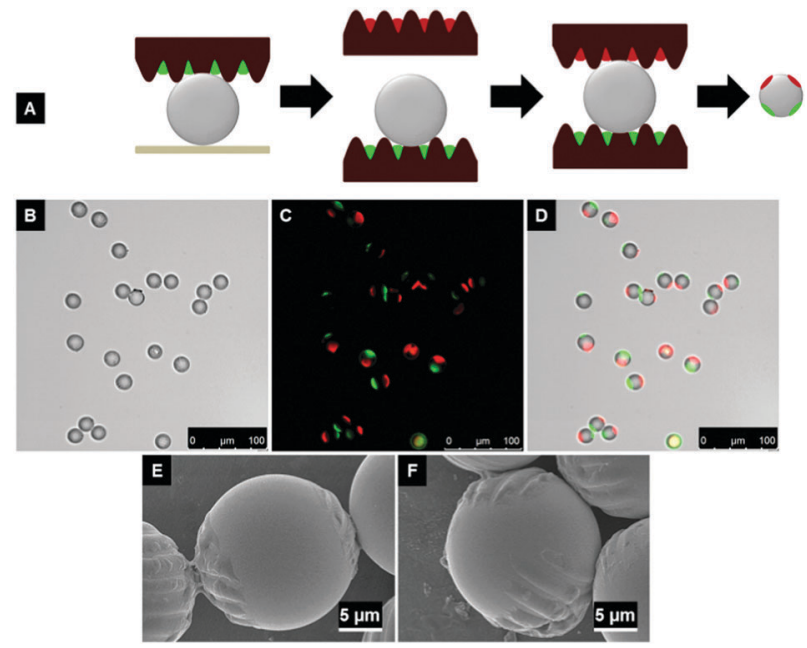

Fig. 4 (A) Schematic depiction of the "sandwich"-intaglio printing process with wrinkles emphasizing the successive printing of both particle hemispheres by using two individual stamps. (B-D) Bright field (B), fluorescence (C) and overlay (D) images of silica particles $(20 \mu \mathrm{m})$ after "sandwich"-intaglio printing with wrinkled stamps $(\lambda=3 \mu \mathrm{m})$. The stamps were loaded with PEI/FITC (green) and PEI/RBITC (red) and the particles were removed by acetone. (E and F) SEM images of silica particles $(20 \mu \mathrm{m})$ after "sandwich"-intaglio printing with wrinkled $\operatorname{stamps}(\lambda=3 \mu \mathrm{m})$. 
with the particles from the glass slide, the other hemisphere is brought into contact with the second stamp, which is loaded with the other fluorescent dye. Subsequently, the stamps have to be separated from each other before the particles can be removed. The particles are now distributed over both stamp surfaces and can be removed from them by ultra-sonication in acetone. For a successful printing with modified PEI, it is not only important which hemisphere of the particles is printed first but also which ink is used.

In Fig. 4B to D, the respective silica particles, now functionalized with PEI-FITC and PEI-RBITC, are presented. The striped structure of the patches cannot be seen easily in the bright field or fluorescence image. However, a successful modification of both hemispheres is observed for almost every particle. Moreover, the striped structure of the printed patches is noticeable from the respective SEM images (Fig. 4E and F) and after changing the focal plane in the fluorescence microscope. In the latter case, we can focus on the two functionalized hemispheres separately, and by that emphasizing the striped structure of the patches for each hemisphere (see the ESI; $\dagger$ Fig. S6).

The "sandwich"-printing also works with smaller $(5 \mu \mathrm{m})$ silica particles. The respective results are presented in the ESI $\dagger$ (Fig. S7).

\section{Conclusion}

In conclusion, we could use intaglio printing with wrinkled stamps loaded with PEI to yield multi-patch particles in one single step. Depending on the particle size and chosen wavelength of the stamp, we could control the number and distance between the patches easily. Moreover, the solvent that is used for removing the particles from the stamp after printing, the ink concentration as well as the stiffness of the stamp influence the printing process and by that, the patch formation. Targeting of all these parameters finally leads to patch sizes and structures, which can be very well tuned.

Finally, the "sandwich"-printing technique was successfully applied for silica particles with a diameter of $20 \mu \mathrm{m}$ as well as, accompanied by a lower reproducibility, $5 \mu \mathrm{m}$. Nonetheless, we found a new way to functionalize particle hemispheres separately while at the same time creating anisotropic patch geometries with different functionalities. By using this straightforward process with wrinkled stamps instead of flat stamps, which was not done before, we achieve a high diversity of combined options for printing on particles concerning the chosen ink, patch geometry, number and distance as well as surface chemistry.

\section{Conflicts of interest}

There are no conflicts to declare.

\section{References}

1 S. Jiang, Q. Chen, M. Tripathy, E. Luijten, K. S. Schweizer and S. Granick, Adv. Mater., 2010, 22, 1060.
2 Y. Wang, Y. Wang, D. R. Breed, V. N. Manoharan, L. Feng, A. D. Hollingsworth, M. Weck and D. J. Pine, Nature, 2012, 491, 51.

3 K. J. Lee, J. Yoon and J. Lahann, Curr. Opin. Colloid Interface Sci., 2011, 16, 195.

4 A. Walther and A. H. E. Muller, Chem. Rev., 2013, 113, 5194.

5 Z. Zhang and S. C. Glotzer, Nano Lett., 2004, 4, 1407.

6 S.-H. Kim, G.-R. Yi, K. H. Kim and S.-M. Yang, Langmuir, 2008, 24, 2365.

7 K. H. Roh, D. C. Martin and J. Lahann, Nat. Mater., 2005, 4, 759.

8 T. Nisisako, T. Torii, T. Takahashi and Y. Takizawa, $A d v$. Mater., 2006, 18, 1152.

9 L. Hong, S. Jiang and S. Granick, Langmuir, 2006, 22, 9495. 10 R. Brüx, S. Hiltl, V. Schröder, C. von Essen and A. Böker, Part. Part. Syst. Charact., 2014, 31, 871.

11 H. Bao, W. Peukert and R. Klupp Taylor, Adv. Mater., 2011, 23, 2644.

12 G. Zhang, D. Wang and H. Möhwald, Nano Lett., 2005, 5, 143.

13 C. E. Snyder, A. M. Yake, J. D. Feick and D. Velegol, Langmuir, 2005, 21, 4813.

14 A. B. Pawar and I. Kretzschmar, Langmuir, 2009, 25, 9057.

15 A. B. Pawar and I. Kretzschmar, Macromol. Rapid Commun., 2010, 31, 150.

16 T. Kaufmann, M. T. Gokmen, C. Wendeln, M. Schneiders, S. Rinnen, H. F. Arlinghaus, S. A. Bon, F. E. Du Prez and B. J. Ravoo, Adv. Mater., 2011, 23, 79.

17 T. Tigges, D. Hoenders and A. Walther, Small, 2015, 11, 4540. 18 L. Sanchez, P. Patton, S. M. Anthony, Y. Yi and Y. Yu, Soft Matter, 2015, 11, 5346.

19 S. Sagebiel, L. Stricker, S. Engel and B. J. Ravoo, Chem. Commun., 2017, 53, 9296-9299.

20 A. Kumar and G. M. Whitesides, Appl. Phys. Lett., 1993, 63, 2002.

21 B. J. Ravoo, J. Mater. Chem., 2009, 19, 8902.

22 A. Perl, D. N. Reinhoudt and J. Huskens, Adv. Mater., 2009, 21, 2257.

23 S. Jiang and S. Granick, Langmuir, 2009, 25, 8915.

24 Y. Xia, M. Mrksich, E. Kim and G. M. Whitesides, J. Am. Chem. Soc., 1995, 117, 9576.

25 J. S. Hovis and S. G. Boxer, Langmuir, 2000, 16, 894.

26 Y. Gao and Y. Yu, Langmuir, 2015, 31, 2833.

27 A. Bernard, J. P. Renault, B. Michel, H. R. Bosshard and E. Delamarche, Adv. Mater., 2000, 12, 1067.

28 H. Li, D.-J. Kang, M. G. Blamire and W. T. S. Huck, Nano Lett. , 2002, 2, 347.

29 S. A. Lange, V. Benes, D. P. Kern, J. K. H. Hörber and A. Bernard, Anal. Chem., 2004, 76, 1641.

30 V. Santhanam and R. P. Andres, Nano Lett., 2003, 4, 41.

31 C. Wendeln and B. J. Ravoo, Langmuir, 2012, 28, 5527.

32 D. I. Rozkiewicz, D. Jańczewski, W. Verboom, B. J. Ravoo and D. N. Reinhoudt, Angew. Chem., Int. Ed., 2006, 45, 5292.

33 T. Kaufmann, C. Wendeln, M. T. Gokmen, S. Rinnen, M. M. Becker, H. F. Arlinghaus, F. Du Prez and B. J. Ravoo, Chem. Commun., 2013, 49, 63.

34 P. Seidel and B. J. Ravoo, Macromol. Chem. Phys., 2016, 217, 1467. 
35 D. I. Rozkiewicz, B. J. Ravoo and D. N. Reinhoudt, Langmuir, 2005, 21, 6337.

36 C. Wendeln, A. Heile, H. F. Arlinghaus and B. J. Ravoo, Langmuir, 2010, 26, 4933.

37 C. Wendeln, S. Rinnen, C. Schulz, H. F. Arlinghaus and B. J. Ravoo, Langmuir, 2010, 26, 15966.

38 T. Kaufmann, M. T. Gokmen, S. Rinnen, H. F. Arlinghaus, F. Du Prez and B. J. Ravoo, J. Mater. Chem., 2012, 22, 6190.

39 M. Pretzl, A. Schweikart, C. Hanske, A. Chiche, U. Zettl, A. Horn, A. Böker and A. Fery, Langmuir, 2008, 24, 12748.

40 A. Horn, S. Hiltl, A. Fery and A. Böker, Small, 2010, 6, 2122. 41 J. Genzer and J. Groenewold, Soft Matter, 2006, 2, 310.
42 A. Schweikart and A. Fery, Mikrochim. Acta, 2009, 165, 249.

43 A. Schweikart, A. Horn, A. Böker and A. Fery, Controlled Wrinkling as a Novel Method for the Fabrication of Patterned Surfaces, in Complex Macromolecular, ed. I. Systems, A. H. E. Müller and H.-W. Schmidt, Springer Berlin Heidelberg, Berlin, Heidelberg, 2010, p. 75.

44 Multilayer Thin Films: Sequential Assembly of Nanocomposite Materials, ed. G. Decher and J. B. Schlenoff, Wiley-VCH, Deutschland, 2012.

45 K. Ariga, J. P. Hill and Q. Ji, Phys. Chem. Chem. Phys., 2007, 9, 2319.

46 E. Poptoshev, B. Schoeler and F. Caruso, Langmuir, 2004, 20, 829. 